\title{
Effect of pomegranate seed oil on hyperlipidaemic subjects: a double-blind placebo-controlled clinical trial
}

\author{
Parvin Mirmiran ${ }^{1,2}$, Mohammad Reza Fazeli ${ }^{3}$, Golaleh Asghari ${ }^{1}$, Abbas Shafiee ${ }^{4}$ and Fereidoun Azizi ${ }^{5 *}$ \\ ${ }^{1}$ Obesity Research Center, Research Institute for Endocrine Sciences, Shahid Beheshti University of Medical Sciences, Tehran, Iran \\ ${ }^{2}$ Faculty of Nutrition and Food Technology, National Nutrition and Food Technology Research Institute, Shahid Beheshti \\ University of Medical Sciences, Tehran, Iran \\ ${ }^{3}$ Department of Drug and Food Control, Faculty of Pharmacy, Tehran University of Medical Sciences, Tehran 14174, Iran \\ ${ }^{4}$ Pharmacutical Sciences Research Center, Tehran University of Medicinal Sciences, Tehran 14174, Iran \\ ${ }^{5}$ Endocrine Research Center, Research Institute for Endocrine Sciences, Shahid Beheshti University of Medical Sciences, PO Box \\ 19395-4763, Tehran, Iran
}

(Received 3 September 2009 - Revised 26 January 2010 - Accepted 27 January 2010 - First published online 25 March 2010 )

In vitro and in vivo studies have shown that punicic acid, a type of conjugated fatty acid and the main constituent of pomegranate seed oil (PSO), has anti-atherogenic effects. The present study aimed at determining the effect of PSO treatment on serum lipid profiles. This double-blind placebo-controlled randomised clinical trial included fifty-one hyperlipidaemic subjects, diagnosed according to National Cholesterol Education Program definition, and randomly assigned to the PSO and the control groups. The PSO and placebo groups received $400 \mathrm{mg}$ PSO and placebo twice daily, respectively and were followed up for 4 weeks. Serum concentrations of lipids and lipoproteins were measured before and 4 weeks after intervention. Mean concentration of TAG and the TAG:HDL cholesterol (HDL-C) ratio were significantly decreased after 4 weeks in the PSO group as compared with baseline values (2.75 (SD 1.40) v. 3.45 (SD 1.56) mmol/l, $P=0.009$ and 5.7 (SD 4.6) $v .7 .5$ (SD 5.0), $P=0.031$, respectively). The treatment effect was statistically significant in the PSO group as compared with controls in diminution of cholesterol:HDL-C ratio (5.4 (SD 1.5) v. 5.9 (SD 1.4) $P<0.05$ ) adjusted for baseline values. We found a mean difference for PSO $v$. placebo in HDL-C concentration $(0 \cdot 13 v$. $-0.02 \mathrm{mmol} / \mathrm{l})$ and cholesterol:HDL-C ratio $(-0.42 v .0 .01, P<0.05)$. Serum cholesterol, LDL cholesterol and glucose concentrations and body composition variables remained unchanged. It is concluded that administration of PSO for 4 weeks in hyperlipidaemic subjects had favourable effects on lipid profiles including TAG and TAG:HDL-C ratio.

Pomegranate seed oil: Hyperlipidaemia: Randomised clinical trials

Normalisation of dyslipidaemia, a modifiable risk factor, can prevent or reduce the risk of CVD, which is a leading cause of death worldwide ${ }^{(1,2)}$.

In order to treat dyslipidaemia, different pharmacologic and non-pharmacologic approaches have been used. Statins are the first-line therapy for management of increased LDL cholesterol (LDL-C), despite adverse effects such as gastrointestinal symptoms and muscle aches ${ }^{(3)}$. Although fibrates may decrease serum TAG and increase serum HDL cholesterol (HDL-C), more powerful drugs for normalisation of these lipids need to be investigated.

Conjugated fatty acids are well-known hypolipidaemic agents $^{(4,5)}$ documented for their effect on lipid metabolism ${ }^{(6)}$. PSO (Punica granatum) consists of about $80 \%$ conjugated octadecatrienoic fatty acids, with a high content of 9-cis, 11-trans, 13-cis acid or punicic acid (PA), one of the isomers of conjugated linolenic acid $(\mathrm{CLN})^{(7)}$; animal studies report controversial results for the hypolipidaemic role of $\mathrm{PSO}^{(8,9)}$.
Although Arao et al. ${ }^{(8)}$ showed that PSO supplementation in obese hyperlipidaemic Otsuka-Long-Evans-Tokushima fatty rats reduces TAG accumulation, Yang et al. ${ }^{(9)}$ demonstrated that PSO does not alter serum cholesterol concentration.

Considering the lack of data on the effect of PSO on lipid profiles in human subjects, the present study investigated the effect of PSO treatment on lipid profiles of hyperlipidaemic subjects.

\section{Subjects and methods}

\section{Subjects and study design}

This is a parallel, randomised, double-blind and placebocontrolled study. Subjects were recruited from an endocrine clinic in sequential fashion. Inclusion criteria were age over 20 years, not pregnant or lactating, having no diagnosed

\footnotetext{
Abbreviations: CLA, conjugated linoleic acid; CLN, conjugated linolenic acid; HDL-C, HDL cholesterol; LDL-C, LDL cholesterol; PA, punicic acid.

* Corresponding author: Fereidoun Azizi, fax +98 21 22402463, email azizi@endocrine.ac.ir

This clinical trial was registered at email www.irct.ir as IRCT138711151640N1.
} 
allergy or liver dysfunction, BMI $\leq 35 \mathrm{~kg} / \mathrm{m}^{2}$, serum total cholesterol $>5.2 \mathrm{mmol} / \mathrm{l}$ and serum TAG $>1.65 \mathrm{mmol} / \mathrm{l}$.

Subjects were randomly assigned to one of two groups, the PSO ( $n$ 25) and the placebo ( $n$ 26) groups; randomisation was stratified for lipid reducing drug consumption and blind for the subjects and all investigators, except for the randomiser. At baseline, fasting blood samples were taken, and the subjects were given capsules containing either $400 \mathrm{mg}$ PSO or placebo for 4 weeks; capsules were identically packaged to be indistinguishable. Subjects consumed one capsule of either PSO or placebo twice daily, and they were telephoned weekly to evaluate compliance to medication and to ensure that no side effects occurred. Adherence was determined by capsule count after 4 weeks, and the individuals with $<80 \%$ consumption were excluded.

It was recommended that the subjects keep their regular lifestyle, including diet and physical activity during the course of study. Questionnaires were used to collect demographic data, medical history at baseline, and medication and supplement use at baseline and 4 weeks later. The modifiable activity questionnaire and three $24 \mathrm{~h}$ dietary recalls were collected at baseline and 4 weeks later to assess physical activity and dietary intake. BMI and waist:hip ratio were calculated at baseline and at the end of 4 weeks. Body composition including percentage of lean and fat mass was measured by bioelectrical impedance analysis, using Bodystat 1500MDD at baseline and after 4 weeks.

The present study was conducted according to the guidelines of the Declaration of Helsinki, and all procedures involving human subjects were approved by the ethical committee of the Research Institute for Endocrine Sciences of the Shahid Beheshti University of Medical Sciences. Written informed consent was obtained from all the subjects.

\section{Pomegranate seed oil capsule preparation}

Pomegranate seed oil (PSO) capsules (Pometane) were obtained from Vitane Pharma Gmbh, Inc. (Wolfratshausen, Germany), and the raw material for PSO was obtained from Sarouneh Company (Urmia, Iran). Chemical analysis of PSO showed the major forms of fatty acids to be: PA $(18: 3)$ $72.0 \%$; oil acid $(18: 2) 10.7 \%$; linol acid $(18: 2) 8.4 \%$; palmitic acid (16:0) $4.0 \%$; stearic acid (18:0) $2.6 \%$; trace amounts $(<1 \%)$ of other fatty acids, with total amount of $7.3 \%$ of SFA, $11.7 \%$ of MUFA and $81 \%$ of PUFA; crude PSO contained the antioxidant vitamin $\mathrm{E}$ of $36.90 \mathrm{mg} / 100 \mathrm{~g}$ and was kept at cold temperature to avoid oxidation until formulated in capsules.

\section{Biochemical analysis}

Fasting blood samples were taken after 12.00-14.00 hours overnight fasting at baseline and 4 weeks after intervention, and they were centrifuged to separate serum, for biochemical analysis, conducted at the endocrine research laboratory on the day of blood collection. Fasting plasma glucose was measured by the enzymatic colorimetric method using a glucose oxidation kit. Serum total cholesterol and TAG levels were assayed using enzymatic colorimetric tests with cholesterol esterase and cholesterol oxidise and glycerol phosphate oxidise, respectively; HDL-C was measured by the immunoturbidimetry method after precipitation of apo B containing lipoproteins with phosphotungstic acid (Pars Azmoun Company, Tehran, Iran). LDL-C was calculated from serum total cholesterol, TAG and HDL-C, according to Friedewald's equation $^{(10)}$; it was not calculated when serum TG $>4.52 \mathrm{mmol} / \mathrm{l}$. Insulin and oxidised LDL were measured by

Table 1. Anthropometric, body composition, blood pressure and biochemical variables at baseline and after 4 weeks in the pomegranate seed oil (PSO) and placebo groups $\dagger$

(Mean values and standard deviations)

\begin{tabular}{|c|c|c|c|c|c|c|c|c|c|}
\hline & \multicolumn{4}{|c|}{ PSO (n 23) } & \multicolumn{4}{|c|}{ Placebo (n 22) } & \multirow{3}{*}{$\begin{array}{c}P \text { for treatment } \\
\text { effect } \neq\end{array}$} \\
\hline & \multicolumn{2}{|c|}{ Baseline } & \multicolumn{2}{|c|}{4 weeks } & \multicolumn{2}{|c|}{ Baseline } & \multicolumn{2}{|c|}{4 weeks } & \\
\hline & Mean & $\mathrm{SD}$ & Mean & $\mathrm{SD}$ & Mean & SD & Mean & SD & \\
\hline Cholesterol (mmol/l) & $6 \cdot 79$ & 0.81 & 6.92 & $1 \cdot 22$ & 6.94 & 0.86 & $7 \cdot 12$ & 0.99 & NS \\
\hline $\mathrm{TAG}(\mathrm{mmol} / \mathrm{l})$ & $3 \cdot 45$ & 1.56 & $2 \cdot 75^{\star}$ & 1.40 & 3.63 & 1.79 & $3 \cdot 12$ & 1.59 & NS \\
\hline LDL-C (mmol/l) & 3.90 & 1.09 & $4 \cdot 39$ & 1.48 & 3.95 & $1 \cdot 14$ & $4 \cdot 32$ & $1 \cdot 14$ & NS \\
\hline $\mathrm{HDL}-\mathrm{C}(\mathrm{mmol} / \mathrm{l})$ & $1 \cdot 25$ & 0.39 & $1 \cdot 38$ & 0.44 & $1 \cdot 27$ & 0.23 & 1.25 & 0.26 & $<0.059$ \\
\hline Ox-LDL (mU/l) & 167 & 43 & 170 & 40 & 173 & 39 & 158 & 47 & NS \\
\hline LDL-C:HDL-C ratio & $3 \cdot 37$ & $1 \cdot 24$ & $3 \cdot 41$ & $1 \cdot 18$ & 3.19 & $1 \cdot 12$ & 3.62 & $1 \cdot 25$ & NS \\
\hline Cholesterol:HDL-C ratio & $5 \cdot 87$ & 1.67 & $5 \cdot 45$ & 1.51 & $5 \cdot 58$ & $1 \cdot 21$ & $5 \cdot 89$ & 1.43 & $<0.04$ \\
\hline TAG:HDL-C ratio & 7.49 & 4.95 & $5 \cdot 73^{\star *}$ & 4.55 & 6.92 & 3.75 & 6 & 2.93 & NS \\
\hline Insulin (mU/I) & $7 \cdot 57$ & $3 \cdot 18$ & $7 \cdot 9$ & $3 \cdot 36$ & $7 \cdot 06$ & 2.87 & $6 \cdot 71$ & 2.75 & NS \\
\hline Insulin:glucose ratio & 0.08 & 0.04 & 0.09 & 0.04 & 0.08 & 0.04 & 0.07 & 0.03 & NS \\
\hline HOMA-IR & 1.68 & 0.69 & $1 \cdot 80$ & 0.84 & 1.55 & 0.65 & 1.50 & 0.63 & NS \\
\hline Body fat (\%) & $36 \cdot 6$ & $6 \cdot 8$ & $36 \cdot 1$ & $10 \cdot 0$ & 41.4 & $6 \cdot 4$ & $40 \cdot 0$ & $10 \cdot 9$ & NS \\
\hline Fat mass $(\mathrm{kg})$ & $27 \cdot 2$ & $6 \cdot 6$ & $26 \cdot 8$ & $9 \cdot 1$ & $31 \cdot 7$ & $8 \cdot 6$ & $30 \cdot 8$ & $11 \cdot 1$ & NS \\
\hline Lean mass (kg) & $47 \cdot 0$ & 7.9 & $43 \cdot 4$ & $11 \cdot 2$ & $44 \cdot 0$ & $6 \cdot 1$ & 41.9 & $10 \cdot 7^{\star \star \star}$ & NS \\
\hline Waist:hip ratio & 0.83 & 0.07 & 0.81 & 0.19 & 0.87 & 0.09 & 0.81 & 0.20 & NS \\
\hline BMI $\left(\mathrm{kg} / \mathrm{m}^{2}\right)$ & $27 \cdot 2$ & $3 \cdot 1$ & $27 \cdot 0$ & $3 \cdot 2$ & $28 \cdot 3$ & 3.7 & $30 \cdot 2$ & $4 \cdot 6$ & NS \\
\hline
\end{tabular}

LDL-C, LDL cholesterol; HDL-C, HDL cholesterol; Ox-LDL, oxidised LDL; HOMA-IR, homeostasis model for insulin resistance.

Mean values were significantly different within the groups using paired $t$ test: ${ }^{\star} P<0.009,{ }^{\star \star} P<0.031,{ }^{* \star} P<0.001$.

† All PSO variables were not significant at baseline, in comparison to placebo.

$\ddagger$ Analysis of covariance with 4-week values as dependent variables, baseline values as covariates and treatment group as a fixed factor. 
ELISA kits (Mercodia AB Company, Uppsala, Sweden); insulin resistance was calculated by the homeostasis model for insulin resistance (HOMA-IR) model, using the formula:

HOMA-IR $=(\operatorname{glucose}(\mathrm{mmol} / \mathrm{l}) \times \operatorname{insulin}(\mathrm{mU} / \mathrm{l})) / 22 \cdot 5$.

\section{Statistical method}

To have a clinically significant effect on lowering serum cholesterol $^{(11)}$, we based our sample calculation to detect a difference of $200 \mathrm{mg} / \mathrm{l}$ and an SD of $200 \mathrm{mg} / 1$ in endpoint cholesterol concentrations between the PSO and placebo groups, and we found that a sample size of 21 was sufficient with $95 \%$ CI and $90 \%$ power in each group. Fifty-one hyperlipidaemic subjects were enrolled to compensate for eventual study dropouts.

SPSS (version 16.0; SPSS, Inc., Chicago, IL, USA) was used for all statistical analyses. Differences between the two groups at baseline were tested with Student's $t$ test and the Mann-Whitney test. Paired Student's $t$ test and Wilcoxon ranked test were used to compare the baseline and 4-week values in each group. To distinguish treatment effect between the groups, following adjustment of their baseline values, analysis of covariance test was used.

\section{Results}

Forty-five of fifty-one patients enrolled in our study were eligible for final analysis, as six withdrew and did not provide 4-week blood samples. Mean values for baseline body weight and age were 74.2 (SD 10.0) and 75.7 (SD 12.2) kg, and 51 (SD 9) and 55 (SD 9) years for the PSO and placebo groups, respectively. No significant differences between the groups were seen for age, sex, weight, height, consumption of lipid lowering drugs or $n-3$ supplements and smoking. All the subjects maintained good health throughout the study, without any major adverse events.

A comparison of dietary intake and physical activity variables at baseline and after 4 weeks between the PSO and placebo groups revealed no significant differences. Energy intake and dietary cholesterol decreased in the placebo group during the treatment period $(P<0.05)$.

The effects of PSO on biochemical, anthropometric, body composition and blood pressure variables are given in Table 1; PSO administration resulted in a decrease in serum
TAG concentration (2.75 (SD 1.40) v. 3.45 (SD 1.56) mmol/l, $P<0.01)$ and a non-significant increase in HDL-C concentration (1.38 (SD 0.44) v. 1.25 (SD 0.39) mmol/l, $P=0.081$ ) compared with baseline values; TAG:HDL-C ratio decreased significantly within the PSO group (5.73 (SD 4.55) v. 7.49 (SD 4.95), $P<0.031$ ). Comparison of treatment effects between the groups with baseline values as covariates revealed lower values for cholesterol:HDL-C (5.45 (SD 1.51) v. 5.89 (SD 1.43), $P<0.05$ ) and higher, but non-significant HDL-C concentrations (1.38 (SD 0.44$)$ v. 1.25 (SD 0.26$) \mathrm{mmol} / \mathrm{l}$, $P=0.059)$ in the PSO group as compared with the placebo group, respectively. Other lipid profile variables including cholesterol, LDL-C, oxidised LDL and LDL-C:HDL-C ratio revealed no significant differences within or between the groups. Fat mass, BMI and waist:hip ratio decreased in the PSO group, but not significantly. PSO had no effect on systolic and diastolic blood pressure within or between the groups (data not shown). Means for differences were significant in HDL-C concentration $(0.13 v$. $-0.02 \mathrm{mmol} / \mathrm{l})$ and cholesterol:HDL-C ratio $(-0.42 v .0 \cdot 01)$ when the PSO group was compared with the placebo group $(P<0.05$ for both) (Fig. 1).

\section{Discussion}

In the present study, 4 weeks of PSO treatment decreased serum TAG concentration, TAG:HDL-C ratio and cholesterol:HDL-C ratio in the PSO group, as compared with the placebo group.

Insignificant differences in physical activity levels and daily diets of the subjects throughout the study duration indicate that any changes in variables were probably due to PSO treatment. Since a target of $2.11 \mathrm{mmol} / \mathrm{l}$ or less for serum TAG in elderly women has been proposed ${ }^{(12)}$, the decrease in serum TAG from 3.45 (SD 1.56 ) to 2.75 (SD 1.40 ) $\mathrm{mmol} / 1$ may be clinically valuable. Data indicate that each $\mathrm{mg} / \mathrm{dl}$ increase in HDL-C corresponds to a $2-3 \%$ risk reduction of $\mathrm{CHD}^{(13)}$; hence our HDL-C increase of $50 \mathrm{mg} / \mathrm{l}$ in the present study reflects a $10-15 \%$ risk reduction of CHD. Besides the lipid profile, we investigated TAG:HDL-C, cholesterol:HDL-C and LDL$\mathrm{C}: \mathrm{HDL}-\mathrm{C}$ as lipid-related indices. The TAG:HDL-C ratio predicts CVD and metabolic syndrome ${ }^{(14)}$, explaining its crucial role in treatment guidelines; therefore, $1.8^{\circ}$ reduction of the TAG:HDL-C ratio in the present study is another significant finding. High cholesterol:HDL-C ratios $(\geq 4)$ are also associated with coronary artery disease ${ }^{(15)}$, and we have

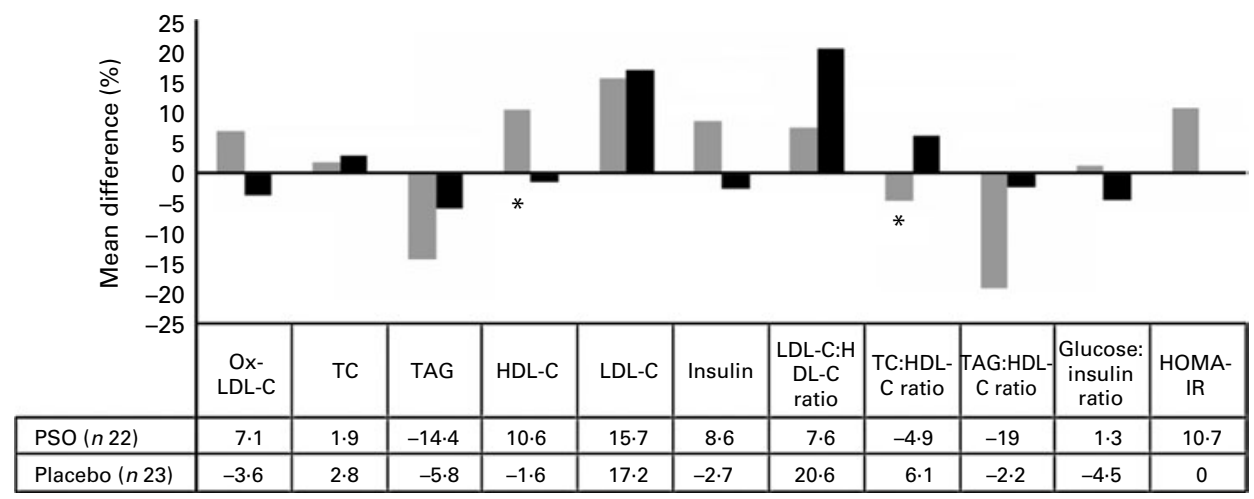

Fig. 1. Mean differences compared with baseline in the pomegranate seed oil (PSO; $\square$ ) and placebo groups ( $\square$ ). Ox-LDL-C, oxidised LDL; TC, total cholesterol; HDL-C, HDL cholesterol, LDL-C, LDL cholesterol; HOMA-IR, homeostasis model for insulin resistance. ${ }^{*} P<0.05$. 
found a significant difference in cholesterol:HDL-C ratio after adjusting for baseline values, in PSO group $v$. placebo group.

A recent study in healthy human subjects reported Trichosanthes kirilowii seeds, naturally occurring sources of PA, had no significant effect on serum lipids ${ }^{(16)}$ contrary to our finding which may be attributable to the pre-existence of hyperlipidaemia in the present study. Current data on the effect of PSO on lipid concentration and metabolism are controversial $^{(8,9,17)}$. Similar to our findings, the liver accumulation of TAG was reduced by PSO administration ${ }^{(8,18)}$; a plausible mechanism, demonstrated in an in vitro study, that 9-cis, 11-trans, 13-cis CLN suppressed cellular synthesis of TAG in HepG2 cells $^{(19)}$.

One study suggests a metabolic pathway for 9-cis, 11-trans, 13-cis CLN is its conversion to 9-cis, 11-trans conjugated linoleic acid (CLA) in a rat model; this conversion coefficient is about $12 \%$ for $\mathrm{PSO}^{(20)}$. Also two recently published articles have demonstrated that PA from T. kirilowii seed oil incorporated into different tissues and serum metabolises to CLA in rats and human subjects ${ }^{(21,22)}$. Hence, the physiological activities of PSO may be attributable to 9-cis, 11-trans CLA derived endogenously or to PA itself. Scientific evidence reveals the inconsistent effects of 9-cis, 11-trans CLA on lipid profiles ${ }^{(23-26)}$, alterations that may occur mostly through the carnitine palmitoyltransferase activity-enzyme involved in fatty acid $\beta$-oxidation, as mitochondrial and peroxisomal carnitine palmitoyltransferase activity is reported to increase with $\mathrm{PSO}^{(18)}$ and $\mathrm{CLN}^{(27)}$; therefore, there is no conclusive evidence to confirm the effect of CLN and CLA on lipid profiles, but it does seem that the isomer type and amount of CLN and CLA could be determining factors.

In the present study, no significant changes were observed in body composition (fat or lean mass), BMI or waist:hip ratio following treatment. Since energy intake, a main contributor to weight gain, did not change during the 4 weeks, decrease in body composition indices is attributable either to PSO or to 9-cis, 11-trans CLA. In vivo studies on weight management and body composition found a reduction on perirenal, epididymal and omental adipose tissues following PA supplementation $^{(8,18)}$.

In the present study, we found no beneficial relationship between insulin profiles and PSO supplementation. A recent animal study showed that PSO reduced the risk of diabetes by lowering glucose and insulin concentrations and insulin sensitivity ${ }^{(28)}$, a result also consistent for specific isomers of CLA, as shown by the Risérus et al. ${ }^{(24)}$ study which reported that administration of $3 \mathrm{~g}$ of 9-cis, 11-trans CLA over 12 weeks yielded a $15 \%$ decrease in insulin sensitivity.

The present study has a few limitations. Serum PSO and CLA concentrations were not measured, which may further have facilitated assessment of compliance of the subjects by measuring PSO metabolites. Our sample size was small, and patients with mixed hyperlipidaemia were enrolled; study duration was 4 weeks and only one blood sample was obtained following intervention. Another limitation might be that we powered our study based on a $200 \mathrm{mg} / 1$ difference in total cholesterol between the PSO and placebo groups; this, however, was not achieved, and despite changes that were observed, one may speculate that the present study is underpowered.
To conclude, the present study found that PSO consumption in hyperlipidaemic subjects did not alter cholesterol and LDL$\mathrm{C}$ but did reduce TAG and TAG:HDL-C ratio during 4-week study period. Further studies of larger samples with longer durations need to confirm the effects of PSO and related mechanisms.

\section{Acknowledgements}

The authors would like to thank the subjects who participated in the present study. The present study was supported by a financial grant (no. 234) from the Research Institute of Endocrine Sciences, Shahid Beheshti University Medical Sciences and Pharmacy and Pharmaceutical Sciences Research Center, Tehran University of Medical Sciences, Iran. None of the authors had any personal or financial conflicts of interest. P. M. was involved in design, implementation and analysis; M. R. F. was involved in preparation of PSO capsules and implementation; G. A. was involved in review of the literature, implementation and analysis; A. S. supervised of technical issues in the preparation of PSO and design; F. A. supervised overall project, design, implementation and analysis.

\section{References}

1. Pearson TA, Blair SN, Daniels SR, et al. (2002) AHA guidelines for primary prevention of cardiovascular disease and stroke: 2002 update: consensus panel guide to comprehensive risk reduction for adult patients without coronary or other atherosclerotic vascular diseases. American Heart Association Science Advisory and Coordinating Committee. Circulation 106, 388-391.

2. Kostis JB (2007) The importance of managing hypertension and dyslipidemia to decrease cardiovascular disease. Cardiovasc Drugs Ther 21, 297-309.

3. Clark LT (2003) Treating dyslipidemia with statins: the riskbenefit profile. Am Heart J 145, 387-396.

4. Wang YM, Nagao K, Inoue N, et al. (2006) Isomer-specific anti-obese and hypolipidemic properties of conjugated linoleic acid in obese OLETF rats. Biosci Biotechnol Biochem $\mathbf{7 0}$, $355-362$.

5. Wilson TA, Nicolosi RJ, Saati A, et al. (2006) Conjugated linoleic acid isomers reduce blood cholesterol levels but not aortic cholesterol accumulation in hypercholesterolemic amsters. Lipids 41, 41-48.

6. Turpeinen AM, Bärlund S, Freese R, et al. (2006) Effects of conjugated linoleic acid on linoleic and linolenic acid metabolism in man. Br J Nutr 95, 727-733.

7. Lansky EP \& Newman RA (2007) Punica granatum (pomegranate) and its potential for prevention and treatment of inflammation and cancer. J Ethnopharmacol 109, 177-206.

8. Arao K, Wang YM, Inoue N, et al. (2004) Dietary effect of pomegranate seed oil rich in 9cis, 11trans, 13cis conjugated linolenic acid on lipid metabolism in obese, hyperlipidemic OLETF rats. Lipids Health Dis 3, 24.

9. Yang L, Leung KY, Cao Y, et al. (2005) Alpha-linolenic acid but not conjugated linolenic acid is hypocholesterolaemic in hamsters. Br J Nutr 93, 433-438.

10. Friedewald WT, Levy RI \& Fredrickson DS (1972) Estimation of the concentration of low-density lipoprotein cholesterol in plasma, without use of the preparative ultracentrifuge. Clin Chem 18, 499-502. 
11. Law MR, Wald NJ \& Thompson SG (1994) By how much and how quickly does reduction in serum cholesterol concentration lower risk of ischaemic heart disease? BMJ 308, 367-372.

12. Mazza A, Tikhonoff V, Schiavon L, et al. (2005) Triglycerides + high-density-lipoprotein-cholesterol dyslipidaemia, a coronary risk factor in elderly women: the cardiovascular study in the elderly. Intern Med J 35, 604-610.

13. Gotto AM Jr (2002) High-density lipoprotein cholesterol and triglycerides as therapeutic targets for preventing and treating coronary artery disease. Am Heart $J$ 144, S33-S42.

14. Hadaegh F, Khalili D, Ghasemi A, et al. (2009) Triglyceride/ HDL-cholesterol ratio is an independent predictor for coronary heart disease in a population of Iranian men. Nutr Metab Cardiovasc Dis 19, 401-408.

15. Nair D, Carrigan TP, Curtin RJ, et al. (2009) Association of total cholesterol/high-density lipoprotein cholesterol ratio with proximal coronary atherosclerosis detected by multislice computed tomography. Prev Cardiol 12, 19-26.

16. Yaun GF, Wahlqvist ML, Yuan J-Q, et al. (2009) Effect of punicic acid naturally occurring in food on lipid peroxidation in healthy young humans. J Sci Food Agric 89, 2331-2335.

17. Yamasaki M, Kitagawa T, Koyanagi N, et al. (2006) Dietary effect of pomegranate seed oil on immune function and lipid metabolism in mice. Nutrition 22, 54-59.

18. Koba K, Imamura J, Akashoshi A, et al. (2007) Genetically modified rapeseed oil containing cis-9, trans-11, cis-13-octadecatrienoic acid affects body fat mass and lipid metabolism in mice. J Agric Food Chem 55, 3741-3748.

19. Arao K, Yotsumoto H, Han SY, et al. (2004) The 9cis, 11trans, 13 cis isomer of conjugated linolenic acid reduces apolipoprotein B100 secretion and triacylglycerol synthesis in HepG2 cells. Biosci Biotechnol Biochem 68, 2643-2645.
20. Tsuzuki T, Kawakami Y, Abe R, et al. (2006) Conjugated linolenic acid is slowly absorbed in rat intestine, but quickly converted to conjugated linoleic acid. J Nutr 136, 2153-2159.

21. Yuan GF, Sinclair AJ, Xu CJ, et al. (2009) Incorporation and metabolism of punicic acid in healthy young humans. Mol Nutr Food Res 53, 1336-1342.

22. Yuan GF, Yuan JQ \& Li D (2009) Punicic acid from Trichosanthes kirilowii seed oil is rapidly metabolized to conjugated linoleic acid in rats. $J$ Med Food 12, 416-422.

23. Tricon S, Burdge GC, Kew S, et al. (2004) Opposing effects of cis-9, trans-11 and trans-10, cis-12 conjugated linoleic acid on blood lipids in healthy humans. Am J Clin Nutr 80, 614-620.

24. Risérus U, Vessby B, Arnlöv J, et al. (2004) Effects of cis-9, trans-11 conjugated linoleic acid supplementation on insulin sensitivity, lipid peroxidation, and proinflammatory markers in obese men. Am J Clin Nutr 80, 279-283.

25. Tricon S, Burdge GC, Jones EL, et al. (2006) Effects of dairy products naturally enriched with cis-9, trans-11 conjugated linoleic acid on the blood lipid profile in healthy middle-aged men. Am J Clin Nutr 83, 744-753.

26. McLeod RS, LeBlanc AM, Langille MA, et al. (2004) Conjugated linoleic acids, atherosclerosis, and hepatic verylow-density lipoprotein metabolism. Am J Clin Nutr 79, $1169 \mathrm{~S}-1174 \mathrm{~S}$

27. Koba K, Akahoshi A, Yamasaki M, et al. (2002) Dietary conjugated linolenic acid in relation to CLA differently modifies body fat mass and serum and liver lipid levels in rats. Lipids 37, 343-350.

28. McFarlin BK, Strohacker KA \& Kueht ML (2009) Pomegranate seed oil consumption during a period of high-fat feeding reduces weight gain and reduces type 2 diabetes risk in CD-1 mice. Br J Nutr 102, 54-59. 